\title{
An Investigation of the Relationship between Involvement Sites in Vitiligo Patients and Autoimmune Diseases and Hematological Parameters
}

\author{
Ebru KARAGUN \\ Duzce University Medical Faculty, Department of Dermatology, Duzce, Turkey \\ Corresponding Author: Ebru KARAGUN, MD; ORCID ID: 0000-0002-5032-7429; karagunebru@ gmail.com \\ Received 22 September 2019; \\ Accepted 30 September 2019; \\ Published 05 October 2019
}

\begin{abstract}
Aim-Objectives: Vitiligo is an acquired idiopathic disease which progresses with melanocyte destruction and is clinically characterized by depigmented lesions of unknown etiology. Vitiligo may be coexistence with a autoimmune and endocrine disorders. This study examined the sT3, fT4, TSH, Anti-TPO, Anti-Tg, Vitamin B12 and fasting blood glucose (FBG) values, and thrombocyte-to-lymphocyte ratio(TLR), neutrophil-to-lymphocyte ratio(NLR), the mean platelet volume(MPV) the correlation of depigmented lesions with the extent of body involvement sites(IS). Materials and Method: The study enrolled 67 patients aged 0-65 who were diagnosed with non-segmental generalized vitiligo and in whom an increase in lesions had been observed in the last six months. The IS of the lesions in the patients were evaluated as IS $<10 \%$ (1st group), $10 \%-20 \%$ (2nd group), $20 \%-30 \%$ (3rd group), $30 \%-40 \%$ (4th group), $40 \%-50 \%$ (5th group), and $>50 \%$ (6th group). The control group consisted of patients who had presented to the outpatient clinic having had no history of vitiligo detected in themselves nor in their families. Results: No significant correlation was found between IS and sT3, fT4, TSH, Anti-TPO, Anti-Tg, Vit. B12, PBG or MPV. A moderately positive correlation was found between IS and duration $(\mathrm{p}<0.05)$ and a mildly positive correlation between IS and NLR and TLR ( $\mathrm{p}$ $<0.05$ ). Conclusion: This study show that every patient diagnosed with vitiligo, independent of the IS, should undergo examination for autoimmune disease. A mild positive correlation between VTA and NLO-TLO was found to be an indicator of increased inflammation in vitiligo patients as the extent of lesions increased.
\end{abstract}

Keywords: Vitiligo, autoimmune diseases, involvement sites, inflammation

\section{Introduction}

Vitiligo is an acquired depigmentation disease characterized by loss of melanocytes in the skin, the cause of which is unknown. The incidence varies between $0.5 \%$ and $1 \%$ from one society to another. The incidence in India has been reported to be $8.8 \% .^{[1,2]}$ Vitiligo may develop at any age; however, $50 \%$ of diagnosed cases are known to occur in the 10-30-year age range. Various systemic diseases, particularly autoimmune diseases, have been identified in relation to vitiligo. Vitiligo may be associated with endocrinological disorders such as thyroid diseases, type I and type II polyglandular syndrome, Addison's disease, hypo parathyroidism, and type 1 diabetes mellitus (DM). Hematological diseases such as autoimmune hemolytic anemia, pernicious anemia, and hemolytic anemia are also associated with vitiligo. ${ }^{[3]}$

Neutrophil and thrombocyte counts are increased in inflammatory and infective events, whereas the lymphocyte count decreases. Like the neutrophil-to-lymphocyte ratio (NLR), the thrombocyte-tolymphocyte ratio (TLR) has a significant correlation with chronic inflammatory diseases. It has been determined that the mean platelet volume (MPV) plays an important role in immunological and inflammatory events, in which it reflects platelet function and activation. Recent studies have suggested that the MPV, NLR, and TLR may be indicative of systemic inflammation. There may be an increase in their levels in immunological and inflammatory incidences, and they may be associated with prognosis of diseases. ${ }^{[4-6]}$

In the present study, the TLR, NLR, and MPV in patients with vitiligo were compared to those of a control group. The correlation between involvement sites (IS) of the lesions and the variances in fT3, fT4, TSH, Anti-TPO, Anti-Tg, Vitamin B12, fasting blood glucose (FBG), TLR, NLR, and MPV were also investigated.

\section{Methods}

The study was designed according to the criteria specified in the Helsinki Declaration. Approval was obtained from the Duzce University Ethics Committee. This study was conducted between October 2017 and December 2018 in the Department of Dermatology and Venereal Diseases of the Duzce University. The 
study enrolled 67 patients between the ages of 0 and 65 who were diagnosed with non-segmental generalized vitiligo and in whom an increase in lesions had been observed in the last six months. Patients who had not received topical and systemic treatment for the last six months. Involvement sites of the lesions in the patients were evaluated as IS $<10 \%$ (1st group), $10 \%-20 \%$ (2nd group), $20 \%-30 \%$ (3rd group), $30 \%-40 \%$ (4th group), $40 \%-50 \%$ (5th group), and $>50 \%$ (6th group). The prevalence of the IS was calculated using the patients' own palms as $1 \%$. The control group was selected from among patients who had no history of vitiligo in families and who subsequently underwent a whole body Wood's lamp examination in the outpatient clinic and no detect vitiligo lesions. Vitiligo and control patients groups with any systemic, inflammatory or infectious disease or a history of malignancy in any organ were not included in the study. The correlation between the variance of fT3, fT4, TSH, Anti-TPO, Anti-Tg, vitamin B12, PBG and the IS of the lesions was investigated. The TLR, NLR, and MPV values (which may vary in autoimmune diseases) of the patients were compared with those of the control group.
Correlations of the TLR, NLR, and MPV with the IS were also examined.

\section{Statistical Analysis}

Data were analyzed using SPSS version 25. The KolmogorovSmirnov test was used to evaluate the distribution of the data. The Mann-Whitney $U$ and the Kruskal-Wallis tests were applied to analyze the non-parametric data with categorical variables. Pearson's Chi-square test and Fisher's exact test were used to analyze categorical variables and Pearson's correlation analysis was performed on the quantitative data. Unless otherwise stated, $\mathrm{P}$ $<0.05$ was considered significant.

\section{Results}

There was no difference between the patient and control groups regarding age, neutrophil count, lymphocyte count, MPV or TLR $(P>0.05)$. In the patient group, the thrombocyte value was significantly lower, and the NLR was significantly higher than in the control group $(\mathrm{P}<0.05)$. (Table 1$)$

Table 1: Personal data of the patients and the comparison of the MTV, NLR, and TLR of the patient group with those of the control group

\begin{tabular}{|c|c|c|c|c|}
\hline & & $\begin{array}{c}\text { Patient Group Mean. } \pm \text { SS/n } \\
(\%)\end{array}$ & Control Group Mean. \pm SS/n (\%) & $\mathrm{p}$ \\
\hline Age & & $35.2 \pm 16.0$ & $33.6 \pm 15.8$ & 0.457 \\
\hline \multirow[t]{2}{*}{ Gender } & Female & $21(31.3 \%)$ & $32(62.7 \%)$ & \multirow[t]{2}{*}{$<0.001$} \\
\hline & Male & $46(68.7 \%)$ & $19(37.3 \%)$ & \\
\hline Thrombocyte(/uL) & & $267.8 \pm 46.8$ & $292.4 \pm 63.7$ & 0.035 \\
\hline Neutrophil(/uL) & & $4300 \pm 1435.3$ & $3954.9 \pm 1216.6$ & 0.153 \\
\hline Lymphocytes(/uL) & & $2068.7 \pm 730.6$ & $2325.4 \pm 674.9$ & 0.086 \\
\hline MTV & & $8.1 \pm 1.3$ & $8.1 \pm 0.9$ & 0.866 \\
\hline NLR & & $2.6 \pm 2.5$ & $1.8 \pm 0.6$ & 0.008 \\
\hline TLR & & $157.3 \pm 116.2$ & $135.7 \pm 51.0$ & 0.418 \\
\hline
\end{tabular}

According to Table 2, there was no significant correlation between IS and sT3, fT4, TSH, Anti-TPO, Anti-Tg, Vit. B12, PBG, thrombocyte, neutrophil, or MPV values. A moderately positive correlation was found between IS and duration $(\mathrm{P}<0.05)$. There was a mildly negative correlation between IS and lymphocyte levels ( $\mathrm{P}<0.05$ ), while a mildly positive correlation was found between IS and NLR and TLR (P <0.05).

Table 2: Relationship between IS ratios and parameters

\begin{tabular}{|l|l|l|l|}
\hline \multicolumn{2}{|l|}{} & $\mathrm{IS}$ & $\mathrm{p}$ \\
\hline & $\mathrm{r}$ & 0.133 & $\mathrm{n}$ \\
\hline Age & 0.185 & $\mathbf{0 . 0 0 0}$ & 67 \\
\hline Duration of illness & $\mathbf{0 . 4 7 2}{ }^{* *}$ & $\mathbf{6 7}$ \\
\hline fT3 & -0.282 & 0.050 & 49 \\
\hline fT4 & 0.013 & 0.917 & 67 \\
\hline TSH & 0.119 & 0.337 & 67 \\
\hline Anti-TPO & 0.230 & 0.062 & 67 \\
\hline Anti-Tg & 0.121 & 0.331 & 67 \\
\hline Vİtamin B12 & 159 & 0.198 & 67 \\
\hline PBG & -0.091 & 0.470 & 66 \\
\hline Thrombocyte & -0.050 & 0.690 & 67 \\
\hline Neutrophil & -0.028 & 0.820 & 67 \\
\hline Lymphocytes & $\mathbf{- 0 . 2 4 4}^{*}$ & $\mathbf{0 . 0 4 7}$ & $\mathbf{6 7}$ \\
\hline MTV & 0.134 & 0.279 & 67 \\
\hline NLR & $\mathbf{0 . 2 9 8}$ & 67 \\
\hline TLR & $\mathbf{0 . 2 9 2}$ & 67 \\
\hline $\begin{array}{l}\text { IS: Involvement site MTV: Mean thrombocyte volume, NLR: Neutrophil-Lymphocyte ratio, TLR: Thrombocyte-Lymphocyte ratio, PBG: } \\
\text { preprandial blood glucose }\end{array}$ & \\
\hline
\end{tabular}

As shown in Table 3, there was no significant relationship between IS and TSH, fT4, fT3, Vit. B12, Anti-TPO, or Anti-Tg values (P >0.05). Anti-TPO was detected in $22.4 \%$ of the patients and positive Anti-Tg was identified in $16.4 \%$. 
Table 3: Comparison in terms of IS

\begin{tabular}{|c|c|c|c|c|c|}
\hline & & & \multicolumn{2}{|l|}{ IS } & \multirow[t]{2}{*}{$\mathrm{p}$} \\
\hline & & Total & $<50 \mathrm{n} \%(\%)$ & $>50 \% \mathrm{n}(\%)$ & \\
\hline \multirow[b]{3}{*}{ TSH } & Low & $1(1.5 \%)$ & $1(1.7 \%)$ & 0 & \multirow[t]{3}{*}{$>0.999$} \\
\hline & Normal & $61(91 \%)$ & $53(89.8 \%)$ & $8(100 \%)$ & \\
\hline & High & $5(7.5 \%)$ & $5(8.5 \%)$ & 0 & \\
\hline \multirow{3}{*}{ fT4 } & Low & $3(4.5 \%)$ & $3(5.1 \%)$ & 0 & \multirow[t]{3}{*}{$>0.999$} \\
\hline & Normal & $57(85.1 \%)$ & $50(84.7 \%)$ & $7(87.5 \%)$ & \\
\hline & High & $7(10.4 \%)$ & $6(10.2 \%)$ & $7(10.4 \%)$ & \\
\hline \multirow{3}{*}{ fT3 } & Low & $4(8.2 \%)$ & $3(6.8 \%)$ & $1(20.0 \%)$ & \multirow[t]{3}{*}{0.574} \\
\hline & Normal & $34(69.4 \%)$ & $30(68.2 \%)$ & $4(80 \%)$ & \\
\hline & High & $11(22.4 \%)$ & $11(25 \%)$ & 0 & \\
\hline \multirow{3}{*}{ Vit b12 } & Low & $11(16.4 \%)$ & $9(15.3 \%)$ & $2(25.0 \%)$ & \multirow[t]{3}{*}{0.609} \\
\hline & Normal & $53(79.1 \%)$ & $47(79.7 \%)$ & $6(75.0 \%)$ & \\
\hline & High & $3(4.5 \%)$ & $3(5.1 \%)$ & 0 & \\
\hline \multirow[t]{2}{*}{ Anti-TPO } & Negative & $52(77,6 \%)$ & $45(76,3 \%)$ & $7(87.5 \%)$ & \multirow[t]{2}{*}{0,672} \\
\hline & Positive & $15(22,4 \%)$ & $14(23,7 \%)$ & $1(12,5 \%)$ & \\
\hline \multirow[t]{2}{*}{ Anti-Tg } & Negative & $56(83,6 \%)$ & $49(83,1 \%)$ & $7(87.5 \%)$ & \multirow[t]{2}{*}{0,750} \\
\hline & Positive & $11(16.4 \%)$ & $10(16,9 \%)$ & $1(12,5 \%)$ & \\
\hline
\end{tabular}

\section{Discussion}

Vitiligo is an acquired, idiopathic disease characterized by melanocyte destruction and depigmented macules and patches whose etiology is not well known. ${ }^{[10]}$ Vitiligo is divided into nonsegmental vitiligo (NSV) and segmental vitiligo (SV). Nonsegmental vitiligo is the most common type in both children and adults. It is characterized by lesions on the face, body and extremities. It tends to spread in relation to the Koebner phenomenon and may be associated with autoimmune diseases. Segmental vitiligo accounts for approximately $10 \%-20 \%$ of vitiligo cases and is characterized by earlier onset and faster development than NSV. In addition, SV is rarely associated with autoimmune disorders or the Koebner phenomenon and is prone to remain stable. ${ }^{[7-9]}$

Several theories have been proposed as to the etiology of the disease. The most widely accepted attributes it to the destruction of autoimmune melanocytes. ${ }^{[10]}$ Two of the best indicators of the correlation between vitiligo and autoimmunity are the high prevalence of autoantibodies against melanocytes in patients with vitiligo and the incidence of autoimmune disease in $10 \%-15 \%$ of vitiligo patients compared to $1 \%-2 \%$ in the general population. ${ }^{[3]}$ The frequency of comorbid autoimmune disease in patients with vitiligo varies around the world. In Japan, Taiwan, and Turkey, autoimmune diseases were detected in $20.3 \%, 14.4 \%$ and $50 \%$ of vitiligo patients, respectively. ${ }^{[11-13]}$ The most common autoimmune diseases in vitiligo patients are Hashimoto's thyroiditis and Graves' disease, both autoimmune thyroid disorders. Other related autoimmune diseases include alopecia areata, pernicious anemia, rheumatoid arthritis (RA), psoriasis, systemic lupus erythematosus (SLE), atopic dermatitis, Addison's disease, myasthenia gravis, Sjögren's syndrome and insulin-dependent diabetes mellitus (IDDM) ${ }^{[14]}$

Epidemiological studies have shown that the prevalence of thyroid disease, especially autoimmune thyroid disease (AITD), is significantly higher in vitiligo patients than in the general population. The prevalence of AITD was $1 \%$ in the general population but ranged from $1.61 \%$ to $37 \%$ in vitiligo patients. ${ }^{[7]}$
Furthermore, the frequency of AITD varies greatly depending on the population examined. An incidence of $19.4 \%$ was reported in Caucasian patients, $7.4 \%$ in Japanese patients, and $2.4 \%-5.8 \%$ in Chinese patients. ${ }^{[11]}$ In a study of 3,280 patients, Shethet al. reported thyroid peroxidase antibody positivity in about half of the patients, thyroid disease in 287 patients, and type $1 \mathrm{DM}$ in 20 patients. ${ }^{[14]}$ Topal et al. found that $12 \%$ of vitiligo patients had low vitamin B12 levels. ${ }^{[15]}$ A meta-analysis study by Vrijman et al. examining 48 studies published between 1968 and 2012 reported the mean prevalence of thyroid disease, autoimmune thyroid disease, and thyroid-specific autoantibodies as $15.1 \%, 14.3 \%$, and $20.8 \%$, respectively. They also found that this risk of these disorders increased by 1.9, 2.9 and 5.2 in patients with vitiligo. ${ }^{[8]} \mathrm{In}$ the present study, Anti-TPO was detected in $22.4 \%$ of the patients, positive Anti-Tg in $16.4 \%$, and low vitamin B12 levels in $16.4 \%$. Type $1 \mathrm{DM}$ was detected in two patients. The findings of the study are consistent with the literature.

The risk of autoimmune disease increases in vitiligo patients and this increase is positively correlated with age. ${ }^{[8]}$ A significant relationship has been reported between increased age and thyroid diseases. ${ }^{[8,16,17]}$ In a retrospective study, Gill et al. found at least one accompanying autoimmune disease associated with an increase in body surface involvement. ${ }^{[18]}$ The present study investigated the correlation between the prevalence of IS and the fT3, fT4, TSH, Anti-TPO, Anti-Tg, Vitamin B12, and PBG values of nonsegmental generalized vitiligo patients. No correlation was found. In contrast to the results of this study, in the literature, the study by Gitt et al. reported an increase in the risk of autoimmune disease with an increased prevalence of body IS. New studies to be carried out in this direction can clarify the risk of autoimmune disease development with the prevalence of body involvement.

In inflammatory and infective events, neutrophil and thrombocyte counts are increased, while lymphocyte counts are decreased. The TLR, NLR, and MPV are significantly associated with chronic inflammatory diseases and are considered indicators of systemic inflammation. ${ }^{[4-6]}$ Studies have identified an increase in the TLR, NLR, and MPV in patients with SLE-RA-psoriasis. They have reported that these values are important in determining disease 
activity and are closely related to the prognosis of disease. ${ }^{[6,19,20]}$ Solak et al. found a higher NLR in generalized vitiligo patients than in the control group or in focal vitiligo patients. ${ }^{[21]}$ In the present study, no difference was found between the patient and control groups in terms of the TLR or MPV; however, the NLR was found to be significantly higher in the patient group. A mild and positive correlation was found between the involvement of body surface and the NLR and TLR. The positive correlation between the NLR and TLR values and IS may have been indicative of systemic inflammation as the body surface involvement increased. It is also known that the NLR, TLR, and MPV are predictors of mortality and morbidity in cardiovascular diseases. ${ }^{[22]}$ Recent studies have reported that vitiligo is not limited to the skin, but is also associated with metabolic syndrome and insulin resistance. ${ }^{[3,22,23]}$ An increase in the NLR, TLR, and MPV in vitiligo patients may be an indicator of insulin resistance or metabolic syndrome. Vitiligo patients should be examined for autoimmune diseases. A link between thyroid diseases and an increase in the age of vitiligo patients has been shown in previous studies. However, no correlation was found between IS and thyroid diseases or autoimmune diseases in this study. We believe that every patient diagnosed with vitiligo should undergo examination for autoimmune disease independent of the IS. A weak positive correlation between the NLR and TLR and an increase in the IS suggested that the inflammation had increased with the prevalence of the lesions in the vitiligo patients. More comprehensive studies may clarify the relationship between the NLR, TLR, and MPV and the IS in patients with vitiligo.

\section{Acknowledgment}

The authors wish to thank the Duzce University Department of Medical Biochemistry (Duzce, Turkey) for their technical support during the preparation of this manuscript.

\section{References}

1. Lee H, Lee MH, Lee DY, et al. Prevalence of Vitiligo and Associated Comorbidities in Korea. Yonsei Med J. 2015;56(3):719-725.

2. Shah H, Mehta A, Astik B. Clinical and socio demographic study of vitiligo. Indian $\mathrm{J}$ Dermatol Venereol Leprol. 2008;74(6):701

3. Lotti T, D'Erme AM. Vitiligo as a systemic disease. Clin Dermatol. 2014;32(3):430-434.

4. Imtiaz F, Shafique K, Mirza SS, et al. Neutrophil lymphocyte ratio as a measure of systemic inflammation in prevalent chronic diseases in Asian population. Int Arch Med. 2012; 5(1): 2.

5. Briggs C. Quality counts: new parameters in blood cellconting. Int J Lab Hematol. 2009;31(3):277-297

6. Erre GL, Paliogiannis P, Castagna F1, et al. Metaanalysis of neutrophil-to-lymphocyte and platelet-tolymphocyte ratio in rheumatoid arthritis. Eur J Clin Invest. 2019;49(1):e13037.

7. Dahir AM, Thomsen SF. Comorbidities in vitiligo: comprehensive review. Int J Dermatol. 2018;57(10):1157-1164.
8. Erre GL, Paliogiannis P, Castagna F, et al. The prevalence of thyroid disease in patients with vitiligo: a systematic review. Br J Dermatol. 2012;167(6):12241235.

9. Mazereeuw-Hautier J, Bezio S, Mahe E, et al. Segmental and nonsegmental childhood vitiligo has distinct clinical characteristics: a prospective observational study. J Am Acad Dermatol. 2010;62(6):945-949.

10. Silverberg NB: Update on childhood vitiligo. Curr Opin Pediatr. 2010;22(4):445-452.

11. Narita T, Oiso N, Fukai K, et al. Generalized vitiligo and associated autoimmune diseases in Japanese patients and their families. Allergol Int. 2011;60(4):505-508.

12. Chen YT, Chen YJ, Hwang CY, et al. Comorbidity profiles in association with vitiligo: a nationwide population based study in Taiwan. J Eur Acad Dermatol Venereol. 2015;29(7):1362-1369.

13. Akay BN, Bozkir M, Anadolu Y, et al. Epidemiology of vitiligo, associated autoimmune diseases and audiological abnormalities: Ankara study of 80 patients in Turkey. $\mathrm{J}$ Eur Acad Dermatol Venereol. 2010;24(10):1144-1150.

14. Sheth VM, Guo Y, Qureshi AA. Comorbidities associated with vitiligo: a ten year retrospective study. Dermatology. 2013;227(4):311-315.

15. Oguz Topal I, Duman H, Gungor S, et al. Evaluation of the Clinical and Sociodemographic Features of Turkish Patients with Vitiligo. Acta Dermato Venerol Croat. 2016;24(2):124-129.

16. Lazzeri L, Colucci R, Cammi A, et al. Adult onset vitiligo: multivariate analysis suggests the need for a thyroid screening. Biomed Res Int. 2016;2016:8065765

17. Bae JM, Lee JH, Yun JS, et al. Vitiligo and overt thyroid diseases: a nationwide population-based study in Korea. J Am Acad Dermatol 2017;76(5):871-878.

18. Gill L, Zarbo A, Isedeh $\mathrm{P}$, et al. Comorbid autoimmune diseases in patients with vitiligo: A cross-sectional study. J Am Acad Dermatol. 2016;74(2):295-302.

19. Li L, Xia Y, Chen C, et al. Neutrophil-lymphocyte ratio in systemic lupus erythematosus disease: a retrospective study. Int J ClinExp Med. 2015;8(7):11026-11031.

20. Kim DS, Shin D, Lee MS, et al. Assessment of neutrophil to lymphocyte ratio and platelet to lymphocyte ratio in Korean patients with psoriasis vulgaris and psoriatic arthritis. J Dermatol. 2016;43(3):305-310.

21. Solak B, Dikicier BS, Cosansu NC, et al. Neutrophil to lymphocyte ratio in patients with vitiligo. Postepy Dermatol Alergol. 2017;34(5):468-470.

22. Wang $X$, Zhang G, Jiang $X$, et al. Neutrophil to lymphocyte ratio in relationto risk of all-cause mortality and cardiovascular events among patients under going angiography or cardiac revascularization: a meta-analysis of observational studies. Atherosclerosis. 2014;234(1):206-213.

23. Karadag AS, Tutal E, Ertugrul DT, et al. Serum holotranscobalamine, vitamin B12, folic acid and homocysteine levels in patients with vitiligo. Clin Exp Dermatol. 2012; 37(1): 62-64. 